\title{
Über die Adsorption von Zuckerarten durch Tierkohle.
}

\author{
Von
}

R. O. Herzog (nach Versuchen von J. Adler).

(Aus dem chemischen Institut der Technischen Hochschule zu Karlsruhe.)

(Der Redaktion zugegangen am 10. April 1909.)

Im letzten Heft der Biochemischen Zeitschrift berichten P. Rona und L. Michaelis ${ }^{1}$ ) über Adsorption von Dextrose durch Tierkohle. Den Verfassern scheint leichtbegreiflicherweise entgangen zu sein, daß vor einiger Zeit von J. Adler und mir in einer Mitteilung über Vorgänge bei der Gerbung ${ }^{2}$ ) Adsorptionsversuche mit verschiedenen Monosen sowie Biosen und Kohle angeführt wurden. Es sei gestattet, das Ergebnis dieser allerdings nur zur Orientierung dienenden Experimente hier wiederzugeben, da sie an der angeführten Stelle nur unvollständig und zum Teil durch ein Versehen unrichtig reproduziert und kaum andeutungsweise diskutiert wurden.

Als Adsorptionsmittel diente «mit Säure gereinigte Blutkohle» von Merck, die von uns nicht weiter vorbehandelt wurde.

Um zu bestimmen, wann die Adsorption beendet sei, wurden Dextroselösungen mit Tierkohle geschüttelt und zu verschiedenen Zeiten analysiert (s. folgende Tab.).

Man sieht, daß nach 30 Minuten die Adsorption als beendigt betrachtet werden kann, doch wurde bei den weiteren Versuchen stets ein Zeitraum von 4 Stunden unter Schütteln abgewartet.

1) Bd. XVI, S. 489 (1909).

2) Zeitschr. f. Chem. und Ind. d. Kolloide, Bd. II, Suppl., Heft 2 (1908). Vgl. auch J. Adler, Zur Theorie der Gerbung, Dissertation, 1908. - Die Versuche wurden noch vor dem Erscheinen der Habilitationsschrift Freundlichs «Über die Asorption in Lösungen. 1906 begonnen. 


\begin{tabular}{|c|c|c|c|c|}
\hline & & $\begin{array}{l}5 \mathrm{~g} \text { Zucker in } \\
100 \mathrm{ccm} \\
\text { Lösung mit } \\
1 \mathrm{~g} \text { Kohle }\end{array}$ & $\begin{array}{c}5 \mathrm{~g} \mathrm{Zucker} \mathrm{in} \\
100 \mathrm{ccm} \\
\text { Lösung mit } \\
5 \mathrm{~g} \text { Kohle }\end{array}$ & $\begin{array}{l}17,1 \mathrm{~g} \text { Zucker } \\
\text { in } 100 \mathrm{ccm} \\
\text { Lösung mit } \\
5 \mathrm{~g} \text { Kohle }\end{array}$ \\
\hline Drehu & der ursprüngl. Lösung & $6,61^{\circ}$ & $6,57^{\circ}$ & $22,90^{\circ}$ \\
\hline$\gg$ & nach 30 Minuten . & $6,29^{\circ}$ & $6,98^{\circ}$ & $20,94^{0}$ \\
\hline 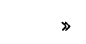 & » 1 Stunde... & $6,28^{\circ}$ & $4,94^{0}$ & $20,95^{\circ}$ \\
\hline s & 3 Stunden . . & $6,28^{\circ}$ & $4,92^{\circ}$ & $20,88^{\circ}$ \\
\hline$>$ & » 30 & - & $4,92^{0}$ & $20,89^{\circ}$ \\
\hline$\triangleright$ & 52 & $6,26^{\circ}$ & - & - \\
\hline
\end{tabular}

Weiter wurde geprüft, ob die Kohle nicht etwa katalytisch Oxydation des Traubenzuckers veranlaßt, indem 4 Stunden lang ein Wasserstoffstrom durch eine $5 \%$ ige Zuckerlösung, der $1 \mathrm{~g}$ Tierkohle zugesetzt war, durchgeleitet wurde. Die Polarisation ergab hierauf einen Drehungswinkel von 6,31 ${ }^{\circ}$, der mit den bei Gegenwart von Luft erhaltenen Werten (vgl. obige Tabelle, 1. Vertikalreihe) innerhalb der Fehlergrenzen identisch ist.

Um festzustellen, ob ein reversibles Gleichgewicht zwischen der von der Kohle absorbierten und der in Wasser gelösten Menge vorliegt, wurde folgender Versuch angestellt. ${ }^{1}$ ) Es wurde eine Zuckerlösung bereitet, deren Drehung nach der Verdünnung mit dem dreifachen Volumen Wasser $8,20^{\circ}$ betrug. Von dieser Lösung wurden

1. $25 \mathrm{ccm}$ mit $25 \mathrm{ccm}$ Wasser und $5 \mathrm{~g}$ Tierkohle versetzt und nach 4 stündigem Schütteln filtriert; das Filtrat mit dem gleichen Volumen Wasser verdünnt, zeigte einen Drehungswinkel von $6,24^{\circ}$;

2. $25 \mathrm{ccm}$ mit $5 \mathrm{~g}$ Tierkohle 3 Stunden geschüttelt, hierauf mit $25 \mathrm{ccm} \mathrm{Wasser} \mathrm{versetzt,} \mathrm{wieder} 3$ Stunden geschüttelt und dann filtriert; das auf die Hälfte verdünnte Filtrat ergab den Drehungswinkel 6,24 ${ }^{\circ}$;

3 . $25 \mathrm{ccm}$ mit $25 \mathrm{ccm}$ Wasser und $2,5 \mathrm{~g}$ Tierkohle ver-

1) Diese Versuche wurden von Herrn Dipl--Ing. A. Polotzky auf meine Bitte ausgeführt.

H. 
setzt und 3 Stunden geschüttelt; nach 3 Stunden wurden wieder $2,5 \mathrm{~g}$ Tierkohle zugesetzt und nach weiterem 3 stündigen Schütteln wurde filtriert; das auf die Hälfte verdünnte Filtrat drehte um 6,21 ${ }^{\circ}$.

Die Drehungswinkel sind also bei allen 3 Versuchen identisch, was die Existenz des Gleichgewichtes beweist.

Die folgenden Tabellen geben nun an, wieviel bei konstantem Volumen $(100 \mathrm{ccm})$ und konstanter Kohlenmenge $(5 \mathrm{~g})$ aus verschiedenen Zuckerkonzentrationen adsorbiert wurde. Ist A die ursprünglich im Wasser gelöste Zuckermenge, und $\mathrm{G}$ die von der Kohle absorbierte, so ist $\mathrm{A}-\mathrm{C}=\mathrm{B}$ die nach der Adsorption in Lösung verbliebene Menge. Zwischen diesen Größen besteht die Bezeichnung $\mathrm{C}=\mathrm{kB}^{\mathrm{n}}$, wo $\mathrm{k}$ und $\mathrm{n}$ für jeden Stoff charakteristische Konstante bedeuten. Nach Biltz ${ }^{1}$ ) ist $\mathrm{n}<1$, nach Freundlich ${ }^{2}$ ) liegt $\mathrm{n}$ zumeist zwischen 0,2 und 0,5 .

Dextrose.

$\mathrm{n}=0,474 ; \mathrm{k}=0,766$.

\begin{tabular}{c|c|c|c|c}
\hline \hline $\mathbf{A}$ & $\mathrm{G}$ & $\begin{array}{c}\mathrm{B} \\
\text { beob. }\end{array}$ & $\begin{array}{c}\mathrm{B} \\
\text { ber. }\end{array}$ & $\Delta$ \\
\hline 9,03 & 1,93 & 7,10 & 7,06 & 0,04 \\
4,38 & 1,31 & 3,07 & 3,13 & 0,06 \\
1,86 & 0,79 & 1,07 & 1,05 & 0,02
\end{tabular}

Lävulose.

$\mathrm{n}=0,539 ; \mathrm{k}=0,338$.

\begin{tabular}{c|c|c|c|c}
\hline A & G & $\begin{array}{c}\text { B } \\
\text { beob. }\end{array}$ & $\begin{array}{c}\text { B } \\
\text { ber. }\end{array}$ & $\Delta$ \\
\hline 8,45 & 1,01 & 7,44 & 7,62 & 0,18 \\
4,22 & 0,66 & 3,56 & 3,49 & 0,07 \\
1,64 & 0,38 & 1,26 & 1,25 & 0,01
\end{tabular}

1) Zeitschrift f. physik. Chem., Bd. XLVIII, S. 618 (1904). (1906).

2) Habilitationsschrift $\mathfrak{u}$. Zeitschr. f. physik. Chem., Bd. LVII, S. 385

Hoppre-Seyler's Zeitschrift f. physiol. Chemie. LX. 
R. 0. Herzog,

Galaktose.

$\mathrm{n}=0,694 ; \mathrm{k}=1,016$.

\begin{tabular}{c|c|c|c|c}
\hline \hline A & G & $\begin{array}{c}\text { B } \\
\text { beob. }\end{array}$ & $\begin{array}{c}\mathrm{B} \\
\text { ber. }\end{array}$ & $\Delta$ \\
\hline 11,13 & 4,01 & 7,12 & 7,18 & 0,06 \\
5,57 & 2,29 & 3,28 & 3,28 & 0,07 \\
2,23 & 1,01 & 1,12 & 1,12 & 0,01
\end{tabular}

Rohrzucker.

$\mathrm{n}=0,127 ; \mathrm{k}=0,106$.

\begin{tabular}{c|c|r|r|c}
\hline \hline A & C & $\begin{array}{c}\text { B } \\
\text { beob. }\end{array}$ & $\begin{array}{c}\text { B } \\
\text { ber. }\end{array}$ & $\Delta$ \\
\hline 17,47 & 1,56 & 15,91 & 14,86 & 1,05 \\
8,73 & 1,44 & 7,29 & 7,82 & 0,53 \\
3,44 & 1,20 & 2,24 & 1,95 & 0,29
\end{tabular}

Maltose.

$\mathrm{n}=0,133 ; \mathrm{k}=1,142$.

\begin{tabular}{c|c|c|c|c}
\hline A & C & $\begin{array}{c}\text { B } \\
\text { beob. }\end{array}$ & $\begin{array}{c}\text { B } \\
\text { ber. }\end{array}$ & $\Delta$ \\
\hline 16,93 & 1,64 & 15,29 & 15,21 & 0,08 \\
8,46 & 1,48 & 6,98 & 7,03 & 0,05 \\
3,39 & 1,26 & 2,13 & 2,12 & 0,01
\end{tabular}

Milchzucker.

$\mathrm{n}=0,135 ; \mathrm{k}=1,138$.

\begin{tabular}{c|c|r|r|c}
\hline A & G & $\begin{array}{c}\text { B } \\
\text { beob. }\end{array}$ & $\begin{array}{c}\text { B } \\
\text { ber. }\end{array}$ & $\Delta$ \\
\hline 17,79 & 1,67 & 16,12 & 16,62 & 0,50 \\
8,89 & 1,48 & 7,41 & 6,82 & 0,59 \\
3,56 & 1,29 & 2,28 & 2,45 & 0,17
\end{tabular}


Im allgemeinen weichen die Beobachtungen sehr wenig von den Werten ab, die sich nach der angeführten Adsorptionsgleichung berechnen, nur bei Rohrzucker und Milchzucker sind die Differenzen größer. Die Werte von $n$ liegen bei den Monosen in den von Freundlich gezogenen Grenzen, bei den Biosen unterhalb derselben, was übrigens auch Freundlich und Losev ${ }^{1}$ ) bei der Adsorption von Farbstoffen durch Kohle fanden. Von erheblichem Interesse erscheint, daß die Werte von $\mathrm{n}$ bei den Monosen recht wesentlich differieren, während sie bei den Biosen sehr nahe beieinander liegen. Dasselbe zeigen die Werte von $\mathbf{k}$. Dieses Verhalten macht wahrscheinlich, daß bei den Stoffen mit geringerem Molekulargewicht die Konstitution hauptsächlich Einfluß auf die Adsorption nimmt; dagegen scheinen die Versuche mit Biosen anzudeuten, daß ähnliche konstituierte Stoffe mit hohem Molekulargewicht einander ziemlich deckende Adsorptionskurven liefern; z. B. schwanken auch die von Freundlich und Losev für Farbstoffe gegenüber verschiedenem Adsorptionsmaterial gefundenen Werte für $\mathbf{n}$ in ziemlich engen Grenzen. Unverständlich wäre dieses Ergebnis nicht, stehen doch Adsorption, Oberflächenspannung und Molekulargewicht in allerdings noch unerkannter Beziehung zu einander. Sollte sich diese ausgesprochene Vermutung bestätigen, so wäre das Ergebnis wohl auch biologisch wichtig.

Schließlich seien noch Versuche angeführt, die mit anderen Mengen (3 g) Tierkohle ausgeführt wurden. Zur Berechnung kann entweder die Gleichung

$$
\frac{\mathrm{C}}{\mathrm{m}}=\mathrm{k} \mathrm{B} \mathrm{n}
$$

dienen, in der $\mathrm{m}$ die Menge des Adsorbens angibt, oder man kann eine Gleichung von Freundlich ${ }^{2}$ ) anwenden, der gefunden hat, daß bei variabeln Mengen des Adsorptionsmittels (m) und konstanter Menge an gelöstem Stoff (A) bei konstantem Volumen die adsorbierte Menge (C) nach der Gleichung

1) Zeitschrift f. physik. Chem., Bd. LIX, S. 284 (1907).

2) 1 . c. 
84 R. O. Herzog, Über Adsorption von Zuckerarten durch Tierkohle.

$$
\lambda=\frac{1}{\mathrm{~m}} \lg \frac{\mathrm{A}}{\mathrm{A}-\mathrm{C}}
$$

berechnet werden kann, wo $\lambda$ eine Konstante darstellt.

Die in den oben mitgeteilten Tabellen angegebenen ursprünglichen Zuckerkonzentrationen (A) sind immer annähernd $1 / 2,1 / 4$ und $1 / 10$ normale. Zum Vergleich diene folgende Versuchsreihe, bei der wieder annäherungsweise $1 / 4$ normale Lösungen angewandt wurden. Zur Berechnung der 4. Kolonne hat die Gleichung (1) (mit den oben für $\mathrm{n}$ und $\mathrm{k}$ mitgeteilten Werten), zur Berechnung der 5. Kolonne die Gleichung (2) (und die oben bei den $1 / 4$ normalen Lösungen angeführten Zahlen, obwohl die ursprünglichen Konzentrationen nicht genau identisch sind) gedient.

\begin{tabular}{l|c|c|c|c|c}
\hline & A & B & $\begin{array}{c}\mathrm{C} \\
\text { beob. }\end{array}$ & $\begin{array}{c}\mathrm{C}_{1} \\
\text { ber. }\end{array}$ & $\begin{array}{c}\mathrm{C}_{2} \\
\text { ber. }\end{array}$ \\
\hline Dextrose . . . . & 4,38 & 3,42 & 0,96 & 0,82 & 0,84 \\
Lävulose... . . & 4,12 & 3,64 & 0,46 & 0,41 & 0,41 \\
Rohrzucker. . . . & 8,45 & 7,63 & 0,82 & 0,86 & 0,86 \\
Maltose . . . . & 8,16 & 7,27 & 0,89 & 0,83 & 0,88
\end{tabular}

